\title{
Detailed Geodetic Technique Procedures for Structural Deformation Monitoring and Analysis
}

\author{
Eteje, S. O. \\ Department of Surveying and Geoinformatics \\ Nnamdi Azikiwe University, Awka, Anambra State, Nigeria \\ E-mail: eteje.sylvester@yahoo.com
}

\begin{abstract}
The understanding of the detailed procedures of the geodetic technique for structural deformation monitoring and analysis is required to enable a reliable and cost-effective monitoring system for the monitoring of engineering structures, be developed. To do this, the different stages that are involved in deformation studies using the geodetic method are explained in detail. As a result, this paper presents detailed geodetic technique procedures for structural deformation monitoring and analysis. Data collection, data processing, result presentation and analysis stages are discussed in detail.
\end{abstract}

Keywords: deformation, monitoring, analysis, least squares, geodetic, technique, epoch.

DOI: $10.7176 / \mathrm{JSTR} / 6-07-02$

\section{Introduction}

The incessant collapse of engineering structures such as bridges, viaducts, dams, high rise buildings and tunnels in various parts of the world has resulted in their (the structures) monitoring for safety purpose. Structural deformation monitoring, is done to detect movement that could lead to collapse and to allow for sufficient warning to successfully evacuate the structure. As it is well known, engineering structures are subject of deformation due to factors such as changes of groundwater level, changes in the bedrock, increase or decrease of weight, changes of the material properties as a result of changes in temperature, ageing or outside influences, tectonic phenomena and human activities.

The importance of deformation monitoring cannot be underestimated. Several cases of collapsed structures in Nigeria and various parts of the world have been reported. In 2017, a bungalow with a shop extension collapsed in Lagos, in 2018 a seven-story building collapsed in Port-Harcourt. Also in 2018, a building marked for demolition in Miami, United States, collapsed. In Italy, Morandi Bridge in Genoa, collapsed, which led to the death of 43 people. Abdullahi and Yelwa (2016) also gave several instances of collapsed structures as those of the Ojiani dam in Akoko-Edo, in Edo State, the Tiga dam in Kano State some years ago which led to the loss of properties and displacements of thousands of rural inhabitants and the collapse of buildings in Abuja, Lagos and Ibadan. Others are Highland Towers collapse in Malaysia, Savar building collapse in Bangladesh, Omsk building collapse in Russia, Royal Plaza Hotel collapse in Thailand, Grace Divine School collapse in Haiti, etc. All these could have been averted if there had been a program for monitoring the stability or deformation of these structures, according to Abdullahi and Yelwa (2016). This was as a result of undermining the importance of deformation survey, and not knowing and understanding the procedures for the development of a reliable and cost-effective monitoring system for the monitoring of engineering structures.

Most engineering structures are monitored using the geodetic method such as the digital level for vertical displacement and the total station and Global Positioning System (GPS) for horizontal displacement detection. The selection of the method of measurements depends upon the accuracy requirements for the survey (Abdel-Gawad et al, 2014). The implication of using deformation monitoring method that is not suitable in term of accuracy for the monitoring of a particular engineering structure has a significant effect on the magnitudes and directions of the determined deformations (movements) (Eteje, 2019). According to Engineer Manual (2018), surveying accuracy specifications are meant to ensure detection of a given amount of movement under normal operating condition.

The analysis of the deformations, as well as movements of the structures, is crucial to decide whether a significant movement has taken place between observations epochs. This is because the said 
deformations are often of the same magnitudes as the observations errors. To do this, some statistical analyses have to be carried out to confirm if a significant movement has taken place or not.

To develop a reliable and cost-effective monitoring system for the monitoring of engineering structures, the following procedures are considered: the deformation monitoring scheme must consist of discrete object monitoring points located on the surface of the structure where the movement of the entire structure will be determined, a set of stable control points established which defines the reference frame for the movements (Ebeling, 2014), geodetic observations collected at discrete time intervals, or epochs that describe the relative geometry between the monitoring and control/reference points, a set of coordinates and their accuracy estimated from these observations that describe the state of the monitoring network at each epoch. If data from multiple epochs are available, deformations that occurred between these epochs can be derived from two epoch analysis (Ebeling, 2014).

To understand the detailed procedures for the development of a reliable and cost-effective monitoring system for the monitoring and analysis of engineering structures for safety purpose, this paper presents detailed geodetic technique procedures for structural deformation monitoring and analysis.

\section{The Detailed Procedures for Structural Deformation Monitoring and Analysis}

\subsection{Monitoring Points Located on the Surface of the Structure}

The first step is to select/choose suitable points on the structure where studs would be placed. These points must be selected such that they can be observed from not less than two reference/control points. This is necessary because of the least squares adjustment of the observations. The studs must be placed fixed such that any of their movement would be the displacement of the structure itself.

\subsection{Establishment of Control/Reference Points}

A set of control/reference points has to be established on stable grounds or platforms to define the reference frame for the movements. The control points are usually monumented and assumed to be stable throughout the monitoring period. They are also selected where they will not be disturbed and where observations can easily be taken from them to not less than two monitoring points. The observation of the control stations should be done accurately. It should be carried out relative to a set of first-order controls using either GNSS observation or a high precision total station.

\subsection{Geodetic Observations Collection at Discrete-Time Intervals or Epochs}

Having selected the monitoring points on the structure and established the reference stations around the structure, the next step is to carry out observations from the reference stations to the monitoring points at a specified interval. The interval may either be one month, three months, six months or one year depending on the purpose of the survey and the magnitude and direction of the expected deformation/movement. In each epoch observations, each of the monitoring points must be observed from more than one reference station to allow for redundant observations, as well as least squares adjustment of the epoch observations (see Figures 1a and 1b).

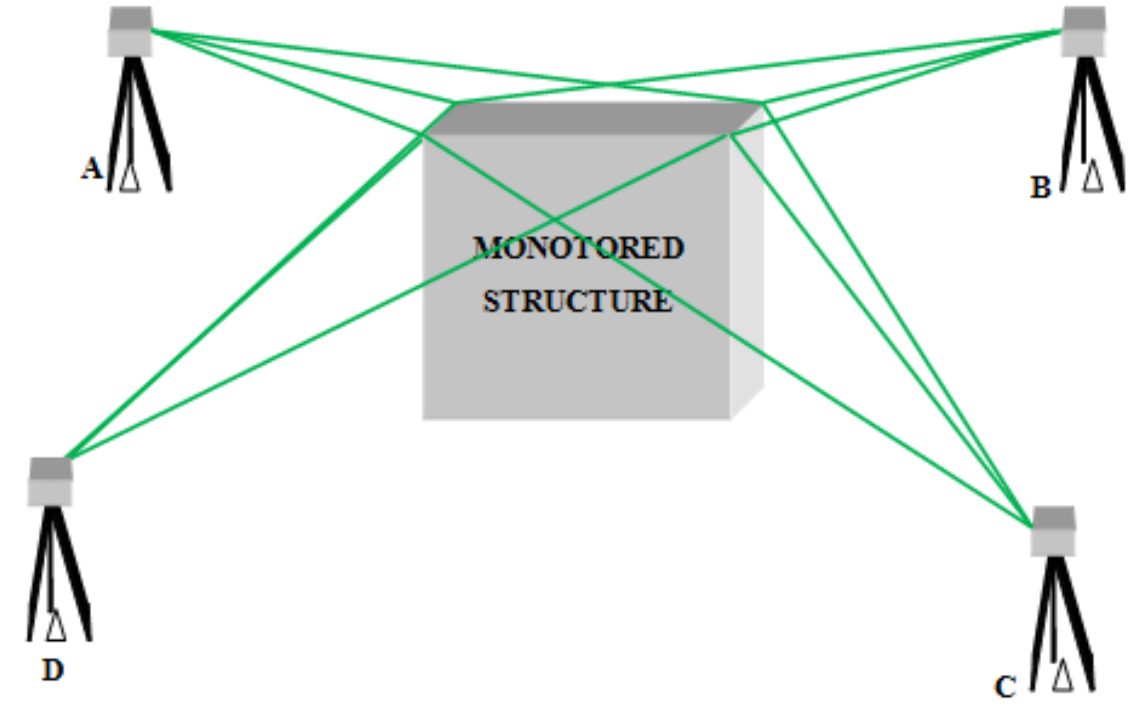

Fig. 1a: Taking Total Station Observations from the Reference Stations to the Monitoring Points 

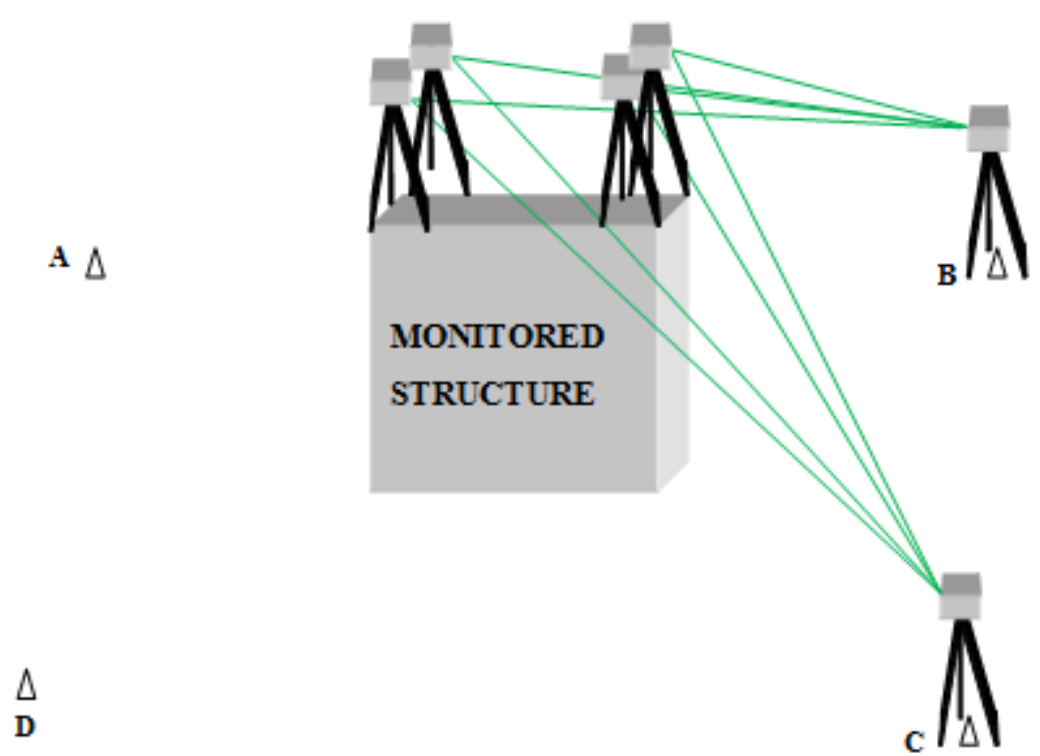

Fig. 1b: GNSS Observation of the Monitoring Points Relative to the Control Stations

\subsection{Processing of Each Epoch Observations}

Each epoch observations are processed and adjusted to obtain: the adjusted coordinates and heights of the monitoring points, the standard errors of the positions and heights of the monitoring points, the error ellipses of each of the processed positions, heights confidence intervals, the a posteriori variance and a posteriori standard error of the observations, the trace of the variance-covariance matrix, Test of the Variance of a Probability Distribution (Chi-Square Test), Redundancy Number, Outlier Detection, Residual Distribution (Goodness of Fit Test) and Tau Statistics. The adjustment is carried out using the observation equation method of the least squares technique.

\subsubsection{Observation Equation Method of Least Squares Adjustment}

The observation equation method of least squares adjustment technique is applied in deformation studies to adjust each epoch observations to enable the precision and accuracy of the adjusted positions and heights, as well as those of the observations, be obtained. In the observation equation method, there is a functional relationship between every measurement and certain parameters. Every measurement (observation) can be related to a certain number of parameters by an equation and the number of equations that can be written, therefore, is exactly equal to the number of measurements used in the adjustment. A solution to these equations is obtained by minimizing the sum of the (weighted) squares of residuals (least squares principle) (equation 1). This 'least squares' solution results in direct determination of parameters.

$$
\phi=v_{1}^{2}+v_{2}^{2}+v_{3}^{2}+\ldots+v_{n}^{2}=\sum_{n=1}^{n} v_{i}^{2}=\text { Minimum }
$$

Ono et al. (2014) gave the following functional relationship between the adjusted observations and the adjusted parameters as:

$$
\mathrm{L}_{\mathrm{a}}=\mathrm{F}\left(\mathrm{X}_{\mathrm{a}}\right)
$$

Where, $\mathrm{L}_{\mathrm{a}}=$ adjusted vector of observations and $\mathrm{X}_{\mathrm{a}}=$ adjusted station coordinates. Equation (2) is a linear function and the general observation equation model was obtained.

To make the matrix expression for performing least squares adjustment, an analogy will be made with the systematic procedures. The system of observation equations is presented by matrix notation as (Mishima and Endo 2002, Ono et al, 2018, Eteje and Oduyebo, 2018)

Where,

$$
V=A X-L
$$




$$
\begin{aligned}
& \mathrm{A}=\text { Design Matrix }=\left(\begin{array}{cccc}
a_{11} & a_{12} & \ldots & a_{1 n} \\
a_{21} & a_{22} & \ldots & a_{2 n} \\
\ldots & \ldots & \ldots & \ldots \\
a_{m 1} & a_{m 2} & \ldots & a_{m n}
\end{array}\right) \\
& \mathrm{X}=\text { Vector of Unknowns }=\left(\begin{array}{c}
x_{1} \\
x_{2} \\
\ldots \\
x_{n}
\end{array}\right)
\end{aligned}
$$

$\mathrm{L}=$ Calculated Values $(l o)$ Minus Observed Values $\left(l_{b}\right)=\left(\begin{array}{l}l_{1} \\ l_{2} \\ \ldots \\ l_{m}\end{array}\right)=l_{0}-l_{b}$

$\mathrm{V}=$ Residual Matrix $=\left(\begin{array}{c}v_{1} \\ v_{2} \\ \cdots \\ v_{m}\end{array}\right)$

That is,

$$
\left(\begin{array}{c}
v_{1} \\
v_{2} \\
\ldots \\
v_{m}
\end{array}\right)=\left(\begin{array}{cccc}
a_{11} & a_{12} & \ldots & a_{1 n} \\
a_{21} & a_{22} & \ldots & a_{2 n} \\
\ldots & \ldots & \ldots & \ldots \\
a_{m 1} & a_{m 2} & \ldots & a_{m n}
\end{array}\right)\left(\begin{array}{c}
x_{1} \\
x_{2} \\
\ldots \\
x_{n}
\end{array}\right)-\left(\begin{array}{l}
l_{1} \\
l_{2} \\
\ldots \\
l_{m}
\end{array}\right)
$$

Estimated parameter

$$
X=(N)^{-1}(t)
$$

Where,

$$
\begin{aligned}
& N=\left(A^{T} W A\right)=\text { Normal Matrix } \\
& t=\left(A^{T} W L\right) \\
& N^{-1}=\left(A^{T} W A\right)^{-1}=Q_{X X} \\
& X=\left(A^{T} W A\right)^{-1}\left(A^{T} W L\right)=\text { Estimate } \\
& \text { W }=\text { Weighted Matrix }
\end{aligned}
$$

\subsubsection{Observation Equation}

The application of least squares adjustment technique for the adjustment of observations in deformation studies requires that observation equations should be formed or written. The number of observation equations must be equal to the number of observations collected. Here, observation equations for GPS baseline vectors for GNSS method, distance and azimuth observation equations for total station method and levelling circuit observation equations are detailed.

\subsubsection{Least Squares Adjustment Model for GPS Baseline Vectors}

Ghilani and Wolf (2008) explained that from the known $X, Y$ and $Z$ coordinates of stations $A$ and $B$, and the observed $\Delta X, \Delta Y$ and $\Delta Z$ components, coordinates of new stations $C, D, E$ and $\mathrm{F}$ can be calculated. An adjustment is necessary because redundant observation exists. In applying least squares to the problem, observation equations are written that relate the unknown adjusted coordinates of the new stations $C, D, E$, and $F$ to the observed $\Delta X, \Delta Y$ and $\Delta Z$ values and their residual errors. The following 
observation equations as given by Ghilani and Wolf (2008) are written for the first two baselines (AC and DC) as

$$
\left.\begin{array}{llr}
X_{C} & =X_{A}+\Delta X_{A C}+V_{1} \\
Y_{C} & =Y_{A}+\Delta Y_{A C}+V_{2} \\
Z_{C} & =Z_{A}+\Delta Z_{A C}+V_{3} \\
X_{C}-X_{D} & = & \Delta X_{D C}+V_{4} \\
Y_{C}-Y_{D}= & \Delta Y_{D C}+V_{5} \\
Z_{C}-Z_{D}= & \Delta Z_{D C}+V_{6}
\end{array}\right\}
$$

Ghilani and Wolf (2008) expressed the observations in equation (6) in matrix form as

$$
\begin{aligned}
A & =\left[\begin{array}{cccccc}
1 & 0 & 0 & 0 & 0 & 0 \\
0 & 1 & 0 & 0 & 0 & 0 \\
0 & 0 & 1 & 0 & 0 & 0 \\
1 & 0 & 0 & -1 & 0 & 0 \\
0 & 1 & 0 & 0 & -1 & 0 \\
0 & 0 & 1 & 0 & 0 & -1
\end{array}\right], & X=\left[\begin{array}{c}
X_{C} \\
Y_{C} \\
Z_{C} \\
X_{D} \\
Y_{D} \\
Z_{D}
\end{array}\right], \\
L & =\left[\begin{array}{ll}
X_{A}+\Delta X_{A C} \\
Y_{A}+\Delta Y_{A C} \\
Z_{A}+\Delta Z_{A C} \\
\Delta X_{D C} \\
\Delta Y_{D C} \\
\Delta Z_{D C}
\end{array}\right], & V=\left[\begin{array}{c}
V_{1} \\
V_{2} \\
V_{3} \\
V_{4} \\
V_{5} \\
V_{6}
\end{array}\right]
\end{aligned}
$$

\subsubsection{Distance Observation Equation}

According to Githumbi (2014), the distance observation equation is

$$
\bar{S}_{i j}=S_{i h}^{o}+\varepsilon_{o}
$$

The distance $S_{12}$ between two points $i$ and $j$ can be computed as

$$
S_{i j}^{0}=\left[\left(X_{j}-X_{i}\right)^{2}+\left(Y_{j}-Y_{i}\right)^{2}\right]^{1 / 2}
$$

Equation (8) is not linear with respect to the unknown parameters $\left(X^{\prime} s\right.$ and $\left.Y^{\prime} s\right)$ and therefore cannot be subjected to a least squares process before it is linearized. The linearization is done by the Taylor series expansion of functions only up to the linear terms. Thus, we now get derivatives of $S_{i j}$ with respect to $X_{i}$ $, Y_{i}, X_{j}, Y_{j}$; as

$$
\left.\begin{array}{l}
\frac{d S_{i j}}{d X_{i}}=-\left(\frac{X_{j}^{o}-X_{i}^{o}}{S_{i j}^{o}}\right) \quad \frac{d S_{i j}}{d X_{j}}=\left(\frac{X_{j}^{o}-X_{i}^{o}}{S_{i j}^{o}}\right) \\
\frac{d S_{i j}}{d Y_{i}}=-\left(\frac{Y_{j}^{o}-Y_{i}^{o}}{S_{i j}^{o}}\right) \quad \frac{d S_{i j}}{d Y_{j}}=\left(\frac{Y_{j}^{o}-Y_{i}^{o}}{S_{i j}^{o}}\right)
\end{array}\right\}
$$


Where $S_{i j}^{0}$ is the distance computed from approximate coordinates, $X_{i}^{o}, Y_{i}^{o}, X_{j}^{o}, X_{j}^{o}$.

\subsubsection{Azimuth Observation Equation}

According to Mikhail and Gracie (1981), the azimuth of a line from point $i$ to point $j$ (in that specific direction) is defined as the horizontal angle measured clockwise from the reference meridian to the line. The direction $A_{i j}$ between two points $i$ and $j$ can be computed as (Githumbi, 2014):

$$
A_{i j}^{o}=\tan ^{-1}\left[\frac{X_{j}-X_{i}}{Y_{j}-Y_{i}}\right]
$$

Mikhail and Gracie (1981) explained that the azimuth can have a value anywhere between $0^{\circ}$ and $360^{\circ}$. It is important to make sure that the quadrant of $A_{i j}$ is correctly determined. This means that the sign of both the numerator $X_{j}-X_{i}$ and the denominator $Y_{j}-Y_{i}$ of the tangent function must be taken into account. They further stated that if a computational aid, such as hand calculator or computer, is used and it yields only a positive or negative acute angle $\alpha_{i j}$, an appropriate constant $C$ must be added to $\alpha_{i j}$ to obtain the azimuth $A_{i j}$. That is,

$$
A_{i j}=\alpha_{i j}+C
$$

Ghilani and Wolf (2008) also stated that if the azimuth of the line is between $0^{\circ}$ and $90^{\circ}$, the value of $C$ is $0^{\circ}$. If the azimuth of the line is between $90^{\circ}$ and $270^{\circ}, C$ is $180^{\circ}$ and if the line's azimuth is between $270^{\circ}$ and $360^{\circ}, C$ is $360^{\circ}$. The linearized form of equation (11) is given in Githumbi (2014) as:

$$
\left.\begin{array}{c}
\frac{d A_{i j}}{d X_{i}}=\left(\frac{Y_{j}^{o}-Y_{i}^{o}}{S_{i j}^{O^{2}}}\right) \quad \frac{d A_{i j}}{d Y_{i}}=-\left(\frac{X_{j}^{o}-X_{i}^{o}}{S_{i j}^{O^{2}}}\right) \\
\frac{d A_{i j}}{d X_{j}}=-\left(\frac{Y_{j}^{o}-Y_{i}^{o}}{S_{i j}^{O^{2}}}\right) \quad \frac{d A_{i j}}{d Y_{J}}=\left(\frac{X_{j}^{o}-X_{i}^{o}}{S_{i j}^{O^{2}}}\right)
\end{array}\right\}
$$

\subsubsection{Least Squares Adjustment of Levelling Circuits}

According to Ghilani and Wolf (2008), in adjusting level networks, the observed difference in elevation for each course is treated as one observation containing a single random error. Observation equations are written that relate these observed elevation differences and their residual errors to the unknown elevations of the benchmarks involved. These can then be processed using the least squares adjustment technique given in section 2.4.1 to obtain the adjusted values for the benchmarks and their standard deviations. Ghilani and Wolf (2008) posited that weights in differential levelling are inversely proportional to course lengths. The course length must be in kilometres. Figure 2 shows a levelling network for the least squares adjustment.

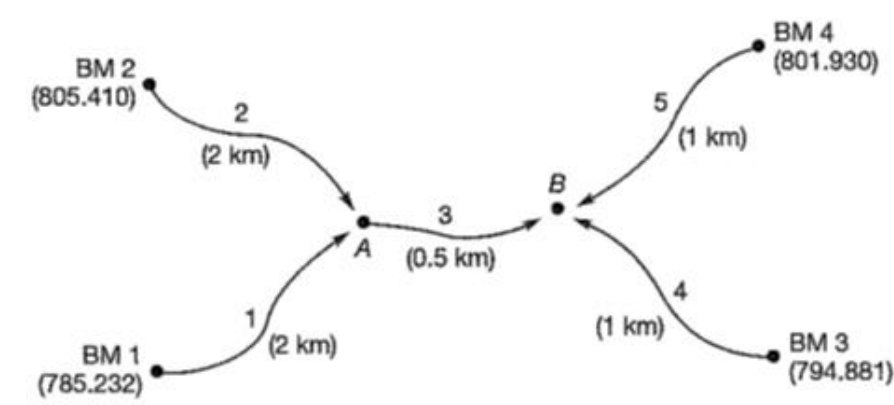

Fig. 2: Levelling Network for Least Squares Adjustment. Source: Ghilani and Wolf (2008) 


\subsubsection{Weight of Observation}

Weight is the worth or reliability of one measurement relative to a standard or another measurement. Measurements with higher precision (smaller standard deviation) should be assigned higher weights and measurements with higher weights should receive smaller corrections after an adjustment. They are relative, and therefore, determined by comparing with another measurement. If the observations have been made with the same precision in each case, it would seem fairly obvious to allot weights proportional to the number of observations in each set. Allan, (2013) explained that weights are assigned to observations so that the weight of observation is proportional to the inverse expected (prior) variance of that observation,

$$
W=\frac{1}{\sigma^{2}}
$$

When a reference variance or variance factor $\left(\sigma_{\circ}^{2}\right)$ is used, weight is given as:

$$
W=\frac{\sigma_{\circ}^{2}}{\sigma^{2}}
$$

The weight of a mean value computed from repeated measurements is proportional to the number of repetitions. The weighted matrix of uncorrelated measurements is a diagonal matrix given as:

$$
W=\left(\begin{array}{cccc}
a_{11} & 0 & 0 & 0 \\
0 & a_{22} & 0 & 0 \\
0 & 0 & \ldots & 0 \\
0 & 0 & 0 & a_{n n}
\end{array}\right)
$$

\subsubsection{Degree of Freedom / Redundant Observation}

Redundant observations are additional measurements which are not required for computing the desired unknown quantities, the parameters. Network redundancy, also known as the number of degree of freedom, is defined as the number of observations minus the number of unknown parameters (Equation 16). The redundant of the observations is useful tools to determine the unknown parameters using the least squares adjustment techniques in geodetic networks (Mohammad, 2016). When there are redundant observations available, traditional coordinate geometry formulae can yield many possible coordinate solutions, depending on which observations are used as input. This inconsistency is remedied by least squares adjustment (Bird, 2009).

$$
r=m-n
$$

Where,

$r=$ Degree of freedom or redundant observations

$\mathrm{m}=$ Number of observations

$\mathrm{n}=$ Number of unknown

\subsubsection{Measure of Accuracy of Adjusted Parameters}

The accuracy information about the estimated parameters is all contained in the variance-covariance matrix, $\sum_{X a}$.

$$
\sum_{X a}=\hat{\sigma}_{\circ}^{2} N^{-1}=\hat{\sigma}_{\circ}^{2}\left(\begin{array}{cccc}
Q_{11} & Q_{12} & \ldots & Q_{1 n} \\
a_{21} & Q_{22} & \ldots & Q_{2 n} \\
\ldots & \ldots & \ldots & \ldots \\
Q_{n 1} & Q_{n 2} & \ldots & Q_{n n}
\end{array}\right)
$$

The accuracy information of the adjusted parameters that contain in the variance-covariance matrix include the:

1. Standard errors of the adjusted positions and heights of the monitoring points.

2. Error ellipses of the adjusted positions.

3. Heights confidence intervals. 


\subsubsection{Standard Errors of the Adjusted Positions and Heights of the Monitoring Points}

The standard errors of the adjusted coordinates and heights of the monitoring points are the measure of the accuracy of the adjusted parameters. Eteje and Oduyebo (2018) gave the model for the computation of the standard errors of the adjusted parameters as:

$$
\hat{\sigma}_{x i}=\hat{\sigma} \cdot \sqrt{Q_{n n}}=\sqrt{\hat{\sigma}_{o}^{2} Q_{n n}}
$$

Where,

$Q_{n n}$ is a diagonal element of the inverse of the normal matrix $\left(N^{-1}\right)$.

\subsubsection{Error Ellipses of the Adjusted Positions}

In surveying and geodesy terms, error ellipse simply is a graphical representation of the marginal standard deviation of the parameters and the covariance between them. Error ellipses are usually computed during adjustment of horizontal or three - dimensional network. They allow a convenient way for the interpretation of the directional station position accuracy. Error ellipses are also used in optimising a network. They can be computed for each adjusted station and between connected stations. The stations' error ellipses are computed from the a posteriori covariance matrix for each station. To compute the relative error ellipse between stations, the a posteriori covariance matrix for the AT station, the TO station and the a posteriori covariance matrix which correlates the two stations are used. Because the error ellipse is a two-dimensional statistical result, the 2D expansion factor is used to scale the semi-major and semiminor axes to the chosen confidence level (in this case, $95 \%$ ).

Mikhail and Gracie (1981) gave the semi-major axis $\sigma_{x^{1}}^{2}$, semi-minor axis $\sigma_{y^{1}}^{2}$ and the orientation of the error ellipse $\theta$ as:

$$
\begin{aligned}
& \sigma_{x^{\mathrm{I}}}^{2}=\frac{\sigma_{x}^{2}+\sigma_{y}^{2}}{2}+\left[\frac{\left(\sigma_{x}^{2}-\sigma_{y}^{2}\right)^{2}}{4}\right]+\sigma_{x y}^{2} \\
& \sigma_{y^{\mathrm{I}}}^{2}=\frac{\sigma_{x}^{2}+\sigma_{y}^{2}}{2}-\left[\frac{\left(\sigma_{x}^{2}-\sigma_{y}^{2}\right)^{2}}{4}\right]+\sigma_{x y}^{2} \\
& \tan 2 \theta=\frac{2 \sigma_{x y}}{\sigma_{x}^{2}-\sigma_{y}^{2}}
\end{aligned}
$$

Mikhail and Gracie, (1981) further pointed out that the variances $\sigma_{x^{1}}^{2}$ and $\sigma_{y^{1}}^{2}$ are the eigenvalues of the covariance matrix of the random vector $\left[\begin{array}{c}X \\ Y\end{array}\right]$.

Mikhail and Gracie, (1981) also stated that the probability of the position given by $\mathrm{X}$ and $\mathrm{Y}$ lies on or within the error ellipse is

$$
\begin{aligned}
& P\left[\frac{X^{2}}{\sigma_{x}^{2}}+\frac{Y^{2}}{\sigma_{y}^{2}} \leq c^{2}\right]=P\left[U \leq c^{2}\right] \\
& =\int_{0}^{c^{2}} \frac{1}{2} e^{-u / 2} d u=1-e^{-c^{2} / 2}
\end{aligned}
$$

Where, $\mathrm{c}=$ Expansion Factor (for 95\%, $\mathrm{c}=2.448$ ).

The probability $P\left[U \leq c^{2}\right]$ is represented by the volume under the bivariate normal density surface within the region defined by the error ellipse. The semi-major axis and semi-minor axis confidence ellipse is determined by 
Semi-major axis confidence ellipse $=\sigma_{x^{I}} c$

Semi minor axis confidence ellipse $=\sigma_{y^{I}} c$

\subsubsection{Height Confidence Interval}

The Height Confidence Interval is an estimate of the precision of the adjusted height (orthometric or ellipsoidal, depending on the network adjustment context), at each station and the relative precision of the height between connected stations. The station height confidence interval is computed from the a posteriori covariance matrix for each station. To compute the relative height confidence interval between connected stations, the a posteriori covariance matrix for the AT station, the TO station and the a posteriori covariance matrix which correlates the two stations are also used. Since the height confidence interval is a one-dimensional result, the $1 \mathrm{D}$ expansion factor (1.959) is used to scale the height confidence interval to the chosen confidence level (95\%). The height confidence interval is determined by multiplying the adjusted height standard deviation by the confidence level (say 95\%) expansion factor (c $=1.959$ for 1D) (Columbus, 2009).

\subsubsection{A Posteriori Variance and a Posteriori Standard Error}

The a posteriori variance which is also called the variance of unit weight and the a posteriori standard error which is also known as the standard error of unit weight are respectively measures of precision and accuracy of a given set of observations. In other words, they are respectively the average, as well as the mean precision and accuracy of a given set of observations. The models for the computation of the a posteriori variance and a posteriori standard error as given in Ameh (2013) are:

1. A Posteriori Variance $\left(\hat{\sigma}_{o}^{2}\right)$

$$
\hat{\sigma}_{o}^{2}=\frac{V^{T} W V}{m-n}=\frac{V^{T} W V}{r}
$$

2. A Posteriori Standard Error $\left(\hat{\sigma}_{o}\right)$

$$
\hat{\sigma}_{o}=\sqrt{\frac{V^{T} W V}{m-n}}=\sqrt{\frac{V^{T} W V}{r}}
$$

Where $V, W$ and $m-n=r$ as respectively given in equations (3), (15) and (16).

\subsubsection{Variance-Covariance of the Adjusted Observations}

The model for the computation of the variance-covariance of the adjusted observations $\left(\sum_{L a}\right)$ is given as:

$$
\sum_{L a}=A \sum_{X a} A^{T}=\hat{\sigma}_{\circ}^{2} A N^{-1} A^{T}
$$

2.4.8 Trace of the Variance-Covariance Matrix

The trace of a square matrix $\mathrm{A}$ is defined as the sum of the diagonal elements of $\mathrm{A}$, written as tr $\mathrm{A}$ or sometimes $\operatorname{tr}(\mathrm{A})$, that is,

$$
\operatorname{tr} \mathrm{A}=\sum a_{i i}
$$

If $\mathrm{A}$ is a covariance matrix, then tr $\mathrm{A}$ is the sum of all variances and can be interpreted as a measure of the overall accuracy of the associated vector of random variates (Caspary, 1988). If two sets of adjusted parameters are given, the one with the smaller trace has better accuracy, hence, the smaller the trace the better the accuracy.

\subsubsection{Test of the Variance of a Probability Distribution (Chi-Square Test)}

Bird (2009) stated that after adjustment, certain statistical hypothesis tests are done to check the quality of the solution and to try to detect the presence of outliers. The confidence level normally used for 
network adjustments is $95 \%$. The corresponding significance level of $5 \%$ means that there is a $5 \%$ chance of making a type I error (Bird, 2009). A type I error is when a correct null hypothesis is rejected.

Under the assumption that a population is normally distributed, we can test the null hypothesis $H_{o}$ that the population variance is $\sigma_{o}^{2}$ against the alternative that it is not $\sigma_{o}^{2}$, using the sample variance $S^{2}$ as the test statistics (Mikhail and Gracie, 1981).

$H_{o}$ is accepted if $S^{2}$ the specific value of $S^{2}$ calculated from the sample lies between $\frac{\chi_{a, r}^{2} \sigma_{o}^{2}}{r}$ and $\frac{\chi_{b, r}^{2} \sigma_{\circ}^{2}}{r}$ that is,

$$
P\left[\frac{\chi_{a, r}^{2} \sigma_{\circ}^{2}}{r}<S^{2}<\frac{\chi_{b, r}^{2} \sigma_{\circ}^{2}}{r}\right]=b-a
$$

otherwise, $H_{o}$ is rejected.

Where,

$$
\begin{aligned}
& \sigma_{o}^{2}=\text { a priori variance } \\
& S^{2}=\hat{\sigma}_{o}^{2}=\text { a posteriori variance } \\
& r=\text { degree of freedom or redundant observation }
\end{aligned}
$$

$1-\alpha$ is the probability that $H_{o}$ is accepted when it is true. Thus, $1-\alpha=b-a$

\subsubsection{Redundancy Number}

The redundancy number for each adjusted observation is yet another statistic that may help to isolate problem areas in the network. For each adjusted observation in the network, a redundancy number is calculated. The redundancy number ranges from 0 to 1 . Observations redundancy numbers are a measure of how close the variance of the residual is to the variance of the observation. If the redundancy number is close to 1 , then the variance of the residual is close to the variance of the observation and the variance of the adjusted observation is close to zero. If the redundancy number is close to zero, then the variance of the residual is close to zero, and the variance of the adjusted observation is close to the variance of the observation (Leick, 1990). Intuitively it is expected that the variance of the residuals and the variance of the observations are close. When this is the case, the noise in the residuals equals that of the observations, and the adjusted observations are determined with high precision. The case where the redundancy number is close to 1 is preferred and it is said that the gain on the adjustment is high. If the redundancy number is close to zero, one expects the noise in the residuals to be small.

The redundancy number of each adjusted observation $\left(r_{i}\right)$ and the average redundancy number of a group of adjusted observations $\left(r_{A v}\right)$ are respectively given as (Leick, 1990)

$$
\begin{aligned}
& r_{i}=q_{i} p_{i} \\
& 0 \leq r_{i} \leq 1 \\
& r_{A v}=\frac{n-R(A)}{n}
\end{aligned}
$$

Where,

$q_{i}=$ Diagonal element of the estimated residual cofactor matrix, $\mathrm{Q}_{\mathrm{v}}$

$p_{i}=$ Weight of the ith observation.

$n-R(A)=$ Degree of freedom or redundant observation.

$R(A)=$ Number of unknown parameters.

$n=$ Number of observations.

\subsubsection{Outlier Detection}

According to Githumbi (2014), an outlier is defined as a residual, which according to some test rule is in contradiction to the reference. This operational definition of an outlier allows for a test strategy and a 
clear statistical concept, but is only a relative definition depending on the selected risk level, the assumed distribution and the test procedure. Despite this fundamental difference between the definition of outliers and gross errors, it is naturally expected that the detected outliers are caused by gross errors.

Whenever outlying residuals are detected, it is necessary to thoroughly check the records of the observations to find out if there are any gross errors. If no gross error can be found, the corresponding observation is detected and if necessary, re-measured. The approach to outlier detection is based on the Gauss Markov Model. The first step is usually a global test. If this global test fails, a procedure to flag erroneous measurements follows i.e. robust estimation.

\subsubsection{Residual Distribution (Goodness of Fit Test)}

The Standardized Residual for an observation is simply the ratio of the adjusted observation residual to the residual standard deviation that is,

$$
\text { Standardized Residual }\left(T_{i}\right)=\frac{\text { Adjusted Observation Residual }\left(v_{i}\right)}{\text { Residual Standard Deviation }\left(\sigma_{v_{i}}\right)}
$$

The Standardized Residual test is one of the better indicators for removing possible outlier observations. If the Standardized Residual exceeds the Standardized Residual Rejection Constant (Tau Statistic), the Standardized Residual value is rejected.

The Standardized Residual is a unitless quantity that allows both angular and linear observations to be compared directly. Probability theory suggests that random errors taken from large samples will tend to be normally distributed about their mean. Graphically, it can be represented by a bell-shaped curve, where the majority of the standardized residuals are expected to be clustered about the midpoint by combining a histogram and the numerical results from a Chi-squared Goodness of Fit" test. All the standardized residuals are grouped into classes about the theoretical mean (zero). Any standardized residual outside the leftmost or rightmost class is tabulated as out of range. Therefore, the closer the histogram approximates the bell-shaped curve, the closer the standardized residuals approach normality.

The Chi-squared Goodness of Fit test is a quantitative measure as to the fit of the histogram to the normal distribution. Columbus (2009) stated that the graphical representation of the residual distribution should be considered in combination with the numerical Chi-square test results before concluding the residuals are or are not normally distributed. Columbus (2009) further stated that even if the test fails, the residuals may be considered normally distributed if they form a bell-shaped curve.

\subsubsection{Tau Statistics}

The Tau statistics are used to determine the critical value, as well as the residual rejection constant in residual distribution. Pope (1976) and Bird (2009) defined Tau statistics in terms of Student's distribution as:

$$
\tau_{v, \alpha}=\frac{t_{v-1, \alpha} \cdot \sqrt{v}}{\sqrt{(v-1)+t_{v-1, \alpha}^{2}}}
$$

Where,

$\tau_{v, \alpha}=$ Critical value from the Tau distribution at redundancy $v$ and significance level $\alpha$

$v=$ Degree of freedom

$\alpha=$ Significance level

$t_{v, \alpha}=$ Critical value from the student's distribution at redundancy $v$ and significance level $\alpha$

According to the rule of the test, $H_{o}$ is rejected if $T_{i}$ is greater than $\tau_{v, \alpha}\left(T_{i}>\tau_{v, \alpha}\right)$ otherwise $H_{o}$ is accepted. Where $T_{i}$ is the standardized residual of each observation.

\subsection{Computation of Magnitude and Direction of Displacement/Movement}

Having processed each epoch observations to obtain the adjusted coordinates of the monitoring points and their respective standard errors, and the mean accuracy of the observations, the next step is to compute the magnitude and direction of the displacement/movement that takes place between observation epochs. The monitoring point displacement, $d$ is computed as the difference in coordinates/heights between the measurement epochs as given by Eteje et al. (2018) is 


$$
d=\left[\begin{array}{l}
d_{x} \\
d_{y}
\end{array}\right]=\left[\begin{array}{l}
x_{c}^{i}-x_{c}^{p} \\
y_{c}^{i}-y_{c}^{p}
\end{array}\right]
$$

Where,

$$
\begin{aligned}
& x_{c}^{i}, y_{c}^{i}=\text { Coordinates of last epoch. } \\
& x_{c}^{p}, y_{c}^{p}=\text { Coordinates of the preceding epoch. }
\end{aligned}
$$

And the horizontal displacement magnitude is computed as (Kaloop and $\mathrm{Li}, 2009$ )

$$
|D|=\sqrt{d^{T} d}
$$

The vertical movement $(d H)$ is computed for each object (monitoring) point as (Eteje et al., 2018):

$$
d H=\sqrt{\left(z_{c}^{i}-z_{c}^{p}\right)^{2}}=\sqrt{(d z)^{2}}
$$

Where,

$$
\begin{aligned}
& z_{c}^{i}=\text { Height of last epoch. } \\
& z_{c}^{p}=\text { Height of preceding epoch. }
\end{aligned}
$$

The direction of movement $(\alpha)$ is computed as:

$$
\tan \alpha_{i}=\frac{d y_{i}}{d x_{i}}
$$

\subsection{Computation of 95\% Confidence Ellipses/Intervals}

The $95 \%$ confidence ellipses/intervals are computed with the standard errors of the adjusted parameters and the $95 \%$ confidence level expansion factor, 1.96. In this, the error associated with the observed monitoring point in two different epochs is computed and multiplied by 1.96. The model for the computation of the $95 \%$ confidence ellipse is given by Beshr and Kaloop (2013) as

$$
e_{n}=1.96 \cdot \sqrt{\sigma_{f}^{2}+\sigma_{i}^{2}}
$$

Where, $\sigma_{f}^{2}$ is the standard error squared in position for the (final) or most recent survey, $\sigma_{i}^{2}$ is the standard error squared in position for the (initial) or reference survey. That is, $\sigma_{f}^{2}=\sigma_{f N}^{2}+\sigma_{f E}^{2}$ and $\sigma_{i}^{2}=\sigma_{i N}^{2}+\sigma_{i E}^{2}$. This implies that,

$$
e_{n}=1.96 \cdot \sqrt{\sigma_{f N}^{2}+\sigma_{f E}^{2}+\sigma_{i N}^{2}+\sigma_{i E}^{2}}
$$

Where,

$$
\begin{aligned}
& \sigma_{f N}^{2}=\text { Standard error squared in northing of the final survey } \\
& \sigma_{f E}^{2}=\text { Standard error squared in easting of the final survey } \\
& \sigma_{i N}^{2}=\text { Standard error squared in northing of the initial survey } \\
& \sigma_{i E}^{2}=\text { Standard error squared in easting of the initial survey }
\end{aligned}
$$

\subsection{Analysis}

This consists of the analysis of the adjusted observations which in turn comprises the analysis of the standard errors of the adjusted positions and heights, error ellipses of the adjusted coordinates, height confidence intervals, a posteriori standard error and a posteriori variance of the adjusted observations, 
the trace of the variance-covariance matric, Chi-square test, redundancy number, outlier detection, the goodness of fit test and Tau statistics. It also consists of two epoch analysis.

\subsubsection{Analysis of the Adjusted Observations}

The standard errors of the adjusted positions/coordinates and heights are analysed based on the computed values. It is a measure of the accuracy of the adjusted positions/heights. The smaller the computed values of the standard errors, the better the accuracy of the adjusted coordinates and heights. It is an indicator of the confidence we have in the adjusted parameters. If the computed values of the standard errors of the adjusted positions and heights are very small, then the confidence in the adjusted parameters is high and they are accepted.

The error ellipses of the adjusted positions are used to present graphically the directional accuracy of the adjusted positions. It consists of the direction of the errors and the computed standard errors of the adjusted coordinates. When computed and plotted, it enables one to infer that the computed error is in a particular direction say north-east, north-west, south-east or south-west as the case may be (see Figure 3).

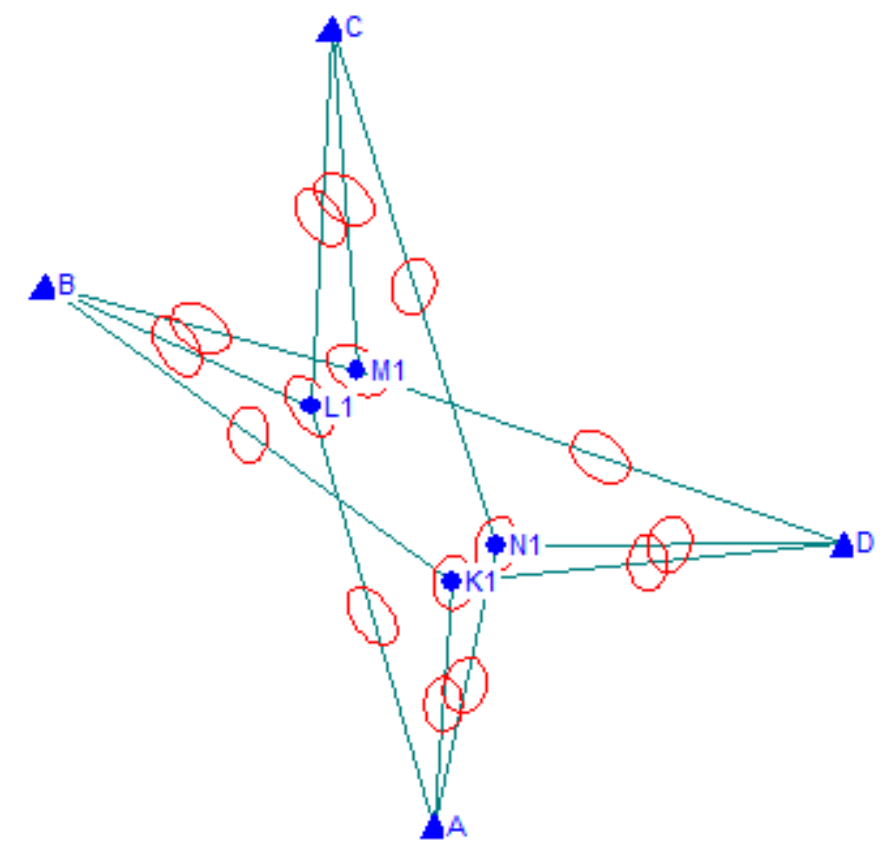

Fig. 3: Plot of Error Ellipses

The height confidence interval is like the positions error ellipses, just that it is one dimension. It is also used to present graphically the accuracy of the adjusted heights. Since height is a vertical component, the heights confidence intervals are plotted vertically (see Figure 4). The smaller the plotted confidence intervals, the higher the accuracy of the adjusted heights. 


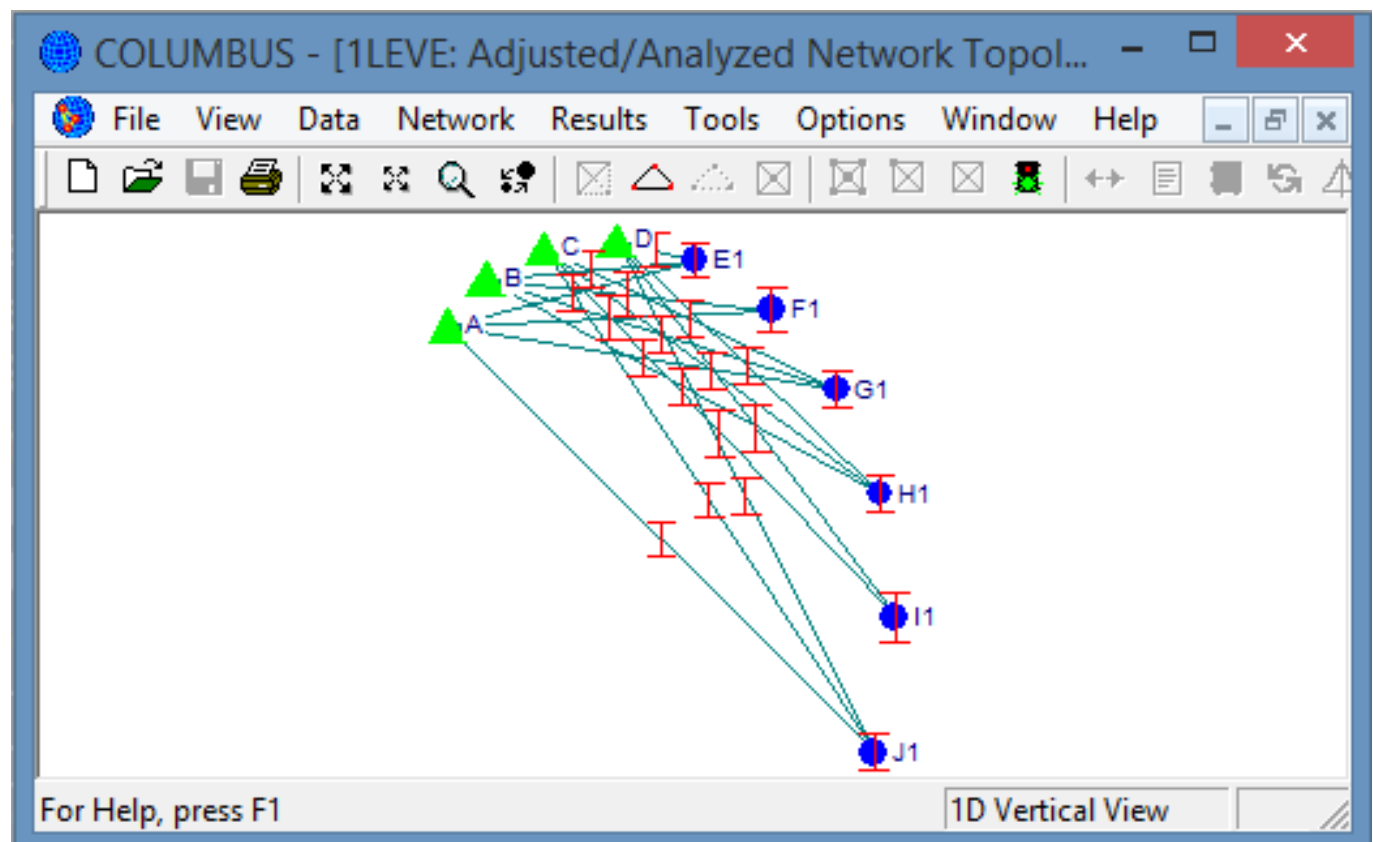

Fig. 4: Plot of Height Confidence Intervals

The a posteriori standard error and a posteriori variance of the adjusted observations are respectively measures of the mean/average accuracy and precision of the adjusted epoch observations. They are computed with the residuals and the degree of freedom, as well as the number of redundant observations. If the values of the computed a posteriori standard error and a posteriori variance are very high, it implies that the accuracy and precision of the epoch observations are low. This means that the residuals, as well as the variations between the adjusted values and the observed values, are very large.

The trace of the variance-covariance matric is another measure of accuracy. It is the sum of the elements of the principal diagonal of the variance-covariance matrix. The principal diagonal of the variancecovariance matrix contains the variations between the adjusted and the observed values. If the differences between the adjusted and the observed values are very small, it implies that the collected observations are very accurate. Their sum is also regarded as the overall accuracy of the adjusted observations. So, the smaller the trace of the variance-covariance matrix, the higher the accuracy of the collected observations. Chi-square test on variance factor is normally carried out to determine how the adjusted result matches the expected result. In this, a priori variance is used which depict the expected variation. In most cases, a priori variance of 1 is used. If the computed a posteriori variance falls within the range of the upper and lower limit as given in equation (28), the set of observations are accepted. But if it falls outside the limit, two things involve, it is either the a priori variance is too large or very small. If the a priori variance is too large, it implies that the accuracy of the collected observations is higher than the expected one. Therefore, the observations are accepted. But if on the other hand, the a priori variance for the survey is very small, it means that very high accuracy is expected. And when this accuracy is less than the expected one, the a posteriori variance will fall outside the range.

Redundancy number is computed to determine the accuracy of individual observation. It ranges from 0 to 1 . If the redundancy number is close to 1 , then the variance of the residual is close to the variance of the observation and the variance of the adjusted observation is close to zero. If the redundancy number is close to zero, then the variance of the residual is close to zero, and the variance of the adjusted observation is close to the variance of the observation (Leick, 1990). Here, if the redundancy number is close to 1 is better. In a group of observations where some of the redundancy numbers are close to 1 and others are close to 0 (zero), if the average redundancy number is close to 1 , the set of observations are accepted.

Outlier detection is done to determine the gross errors in a given set of observations. And this can be done using the goodness of fit test and Tau statistics. In the goodness of fit test, standardized residuals are plotted as a histogram, grouped into classes and combined with a normal distribution, as well as a bell-shaped curve. If all the histogram bars fall within the normal distribution curve, it means that the residuals are normally distributed and the observations that they represent are accepted. But if any of them falls outside the bell-shaped curve, the observations in that class are regarded as outliers as they contain gross errors and are rejected. This test is normally combined with the numerical Chi-square test

20 | P a g e 
(see Figure 5). Columbus (2009) stated that even if the Chi-square test fails, the residuals may be considered normally distributed if they form a bell-shaped curve. Hence the observations are accepted.

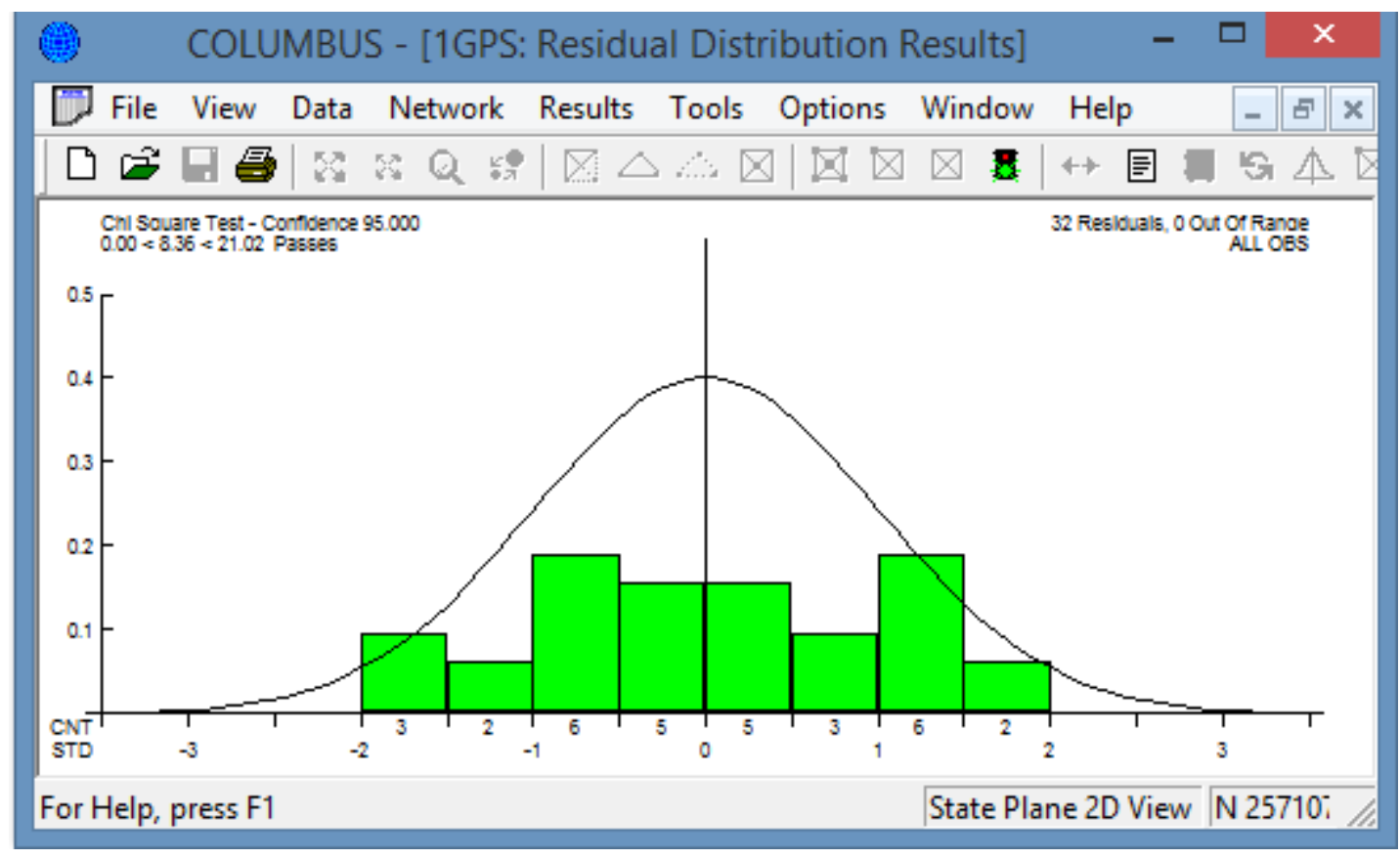

Fig. 5: Goodness of Fit Test

In the Tau statistics, the computed standardized residuals are compared with the standardized residual rejection constant. If the computed standardized residual is less than the standardized residual rejection constant, the observation is accepted, otherwise rejected. Detected gross errors are removed from a given set of observations as their presence affects the overall accuracy of the observations.

\subsubsection{Deformation (Two Epoch) Analysis}

Deformation analysis is often regarded as two epoch analysis. It is done to determine if significant movement/displacement has taken place between any two observation epochs. To do this, the computed displacement for each monitoring point and its corresponding associated error as respectively given in sections 2.5 and 2.6 are compared. If the computed displacement is greater than the associated error, as well as the $95 \%$ confidence interval/ellipse, then a significant movement has taken place between the two measurement epochs. But if on the other hand, the computed displacement is less than the associated error, as well as the $95 \%$ confidence interval/ellipse then significant movement did not take place between the two measurement epochs. Therefore, the computed displacement is regarded as observation/measurement errors.

\section{Conclusion}

In conclusion, this paper has given the step by step procedures to consider developing a reliable and costeffective geodetic monitoring system for the monitoring of engineering structures. The selection of monitoring points on the structure, establishment of reference stations around the structure, taking observations from the reference stations to the monitoring points at a specified interval, processing of each epoch observations, analysis of each epoch observations and two epoch analysis are discussed in detail.

\section{References}

Abdel-Gawad, A. K., Mogahed, Y. M., Mageed, K. M. A. and El-Maghraby, A. M. (2014). Evaluation and Accuracy Assessment of Static GPS Technique in Monitoring of Horizontal Structural Deformations. International Journal of Modern Engineering Research, Vol. 4, No. 6. 
Abdullahi, I. M. and Yelwa, N. A. (2016). Structural Deformation Monitoring Surveys of New Administrative Building of Federal School of Surveying, Oyo - Nigeria. International Journal of Science and Technology, Vol. 6, No.1.

Allan, A. N. (2013). Least Squares Adjustment: Linear and Nonlinear Weighted Regression Analysis. Department of Applied Mathematics and Computer Science/National Space Institute, Technical University of Denmark, Lyngby, Denmark.

Ameh, B. M. (2013). Determination of Components of Deflection of the Vertical of Lobi Area of Makurdi, Benue State, Using GPS (GNSS) and Precise Levelling Observations. Unpublished M.Sc. Thesis of the Department of Surveying and Geo-informatics, Nnamdi Azikiwe University Awka.

Beshr, A. A. and M Kaloop, M. R. (2013). Monitoring Bridge Deformation Using Auto-Correlation Adjustment Technique for Total Station Observations. Positioning, Vol. 4, pp 1-7. DOI:10.4236/pos.2013.41001.

Bird, B. (2009). Analysis of Survey Point Displacements Using Total Station Measurements. Published BSc. Technical Report of the Department of Geomatics Engineering, British Columbia Institute of Technology.

Caspary, W. F. (1988). Concept of Network and Deformation Analysis. Monogram II, School of Surveying, University of New South Wales, Kensington, Australia. ISBN 0-85839-044-2.

Columbus (2009). Columbus 3.8 User Manual. Best Fit Computing Inc. 17490 NW Woodmere Court Beaverton, Oregon 97006 USA.

Ebeling, A. (2014). Ground-Based Deformation Monitoring. Published PhD Thesis of the Department of Geomatics Engineering, University of Calgary, Alberta.

Engineer Manual (2018). Structural Deformation Surveying. US Army Corps of Engineers.

Eteje and Oduyebo (2018). Local Geometric Geoid Models Parameters and Accuracy Determination Using Least Squares Technique. International Journal of Innovative Research and Development, Vol. 7, No. 7, pp 251-257. DOI: 10.24940/ijird/2018/v7/i7/JUL18098.

Eteje and Oduyebo (2018). Understanding Horizontal Geodetic Network Precision and Accuracy Determination Using Least Squares Technique. International Journal for Innovative Research in Multidisciplinary Field, Vol. 4, No. 7, pp 129-135.

Eteje, S. O. (2019). Comparative Analysis of Geodetic Techniques for Monitoring Deformation in Large Structures. Unpublished PhD Dissertation of the Department of Surveying and Geoinformatics, Nnamdi Azikiwe University, Awka.

Eteje, S. O., Ono, M. N. and Oduyebo, F. O. (2018). Monitoring and Analysis of Vertical and Horizontal Deformations of a Large Structure Using Conventional Geodetic Techniques. Journal of Environment and Earth Science, Vol. 8, No. 12, pp 52-61.

Ghilani, C. D. and Wolf, P. W. (2008). Elementary Surveying. An Introduction to Geomatics.12 $2^{\text {th }}$ Edition. Pearson Education, Inc., Prentice Hall, New Jersey, USA.

Githumbi, W. J. (2014). Deformation Monitoring of an Oil Storage Tank by Geodetic Methods. Case Study: Oil Libya, Nairobi Terminal. Published B.Sc. Project of the Department of Geospatial and Space Technology, University Of Nairobi.

Kaloop, M. R. and Li, H. (2009). Monitoring of Bridge Deformation Using GPS Technique. KSCE Journal of Civil Engineering, Vol. 13, No. 6, pp 423-431. DOI: 10.1007/s12205-009-0423-y.

Leick, A. (1990). GPS Satellite Surveying. John Wiley and Sons. 
Mikhail, E. M. and Gracie, G. (1981). Analysis and Adjustment of Survey Measurements. Van Nostrand Reinhold Company, New York.

Mishima, K. and Endo, K. (2002). The Method of the Design for Survey Network by Q Matrices. Proceedings of the 7th International Workshop on Accelerator Alignment, SPring.

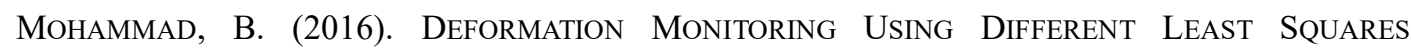
Adjustment Methods: A Simulated Study. KSCE JOURNAL OF CIVIL ENGINEERING, Vol. 20 No. 2 PP 855-862. DOI 10.1007/s12205-015-0454-5

Ono, M. N., Agbo, J. A., Ijioma, D. I. and Chubado, M. (2014). Establishment of Baseline Data for Monitoring of Deformation of Murtala Mohammed Bridge (MMB) Lokoja Kogi State, Using GPS. International Journal of Science and Technology, Vol. 4 No.5, pp 86-92.

Ono, M. N., Eteje, S. O. and Oduyebo, F. O. (2018). Comparative Analysis of DGPS and Total Station Accuracies for Static Deformation Monitoring of Engineering Structures. IOSR Journal of Environmental Science, Toxicology and Food Technology (IOSR-JESTFT), Vol. 12, No. 6, pp 1929.

Pope, A. J. (1976). The Statistics of Residuals and the Detection of Outliers. NOAA Technical Report, NOS 65 NGS 1. Washington, D.C.: U.S. Department of Commerce. In Bird, B. (2009). Analysis of Survey Point Displacements Using Total Station Measurements. Published BSc. Technical Report of the Department of Geomatics Engineering, British Columbia Institute of Technology. 